INPLASY

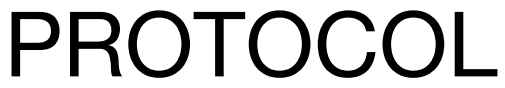

To cite: Yang et al. Prediction Power of Radiomics in Early Recurrence of Hepatocellular Carcinoma: A Systematic Review and Meta-Analysis. Inplasy protocol 202210099. doi:

10.37766/inplasy2022.1.0099

Received: 19 January 2022

Published: 19 January 2022

Corresponding author: Wenbin Ji

wb.j@163.com

Author Affiliation:

Taizhou Hospital of Zhejiang Province affiliated to Wenzhou Medical University, Taizhou, 31800 Zhejiang, China.

Support: No founding.

Review Stage at time of this submission: Data analysis Completed but not published.

\section{Prediction Power of Radiomics in Early Recurrence of Hepatocellular Carcinoma: A Systematic Review and Meta-Analysis}

Yang, J1; You, S2; Zhang, L3; Zhang, H4; Zhang, B5; Dong, X6; Pan, W7; Duan, S8; Ji, W9.

Review question / Objective: Whether radiomics has clinical value for the prediction of early recurrence of HCC?

Condition being studied: Radiomics is an emerging subject of medical science, which has rapidly grown as a reliable method for medical image analysis. It can extract and evaluate quantitative features from traditional medical images using computer algorithms to capture tumor heterogeneity, which is more accurate and efficient than human vision. Investigators employ machine and deep learning techniques to process the extracted quantitative image features, and then use these data to build radiomic models to assist the diagnostic process. In the field of HCC, some studies have suggested that a radiomic signature is a potential biomarker for predicting overall survival and tumor recurrence.

INPLASY registration number: This protocol was registered with the International Platform of Registered Systematic Review and Meta-Analysis Protocols (INPLASY) on 19 January 2022 and was last updated on 19 January 2022 (registration number INPLASY202210099).

Conflicts of interest:

None declared.

\section{INTRODUCTION}

Review question / Objective: Whether radiomics has clinical value for the prediction of early recurrence of HCC?
Condition being studied: Radiomics is an emerging subject of medical science, which has rapidly grown as a reliable method for medical image analysis. It can extract and evaluate quantitative features from traditional medical images using computer algorithms to capture tumor 
heterogeneity, which is more accurate and efficient than human vision. Investigators employ machine and deep learning techniques to process the extracted quantitative image features, and then use these data to build radiomic models to assist the diagnostic process. In the field of HCC, some studies have suggested that a radiomic signature is a potential biomarker for predicting overall survival and tumor recurrence.

\section{METHODS}

Search strategy: A comprehensive literature search for potentially relevant articles was conducted in the embase, Web of Science, and PubMed. The following keywords were used: (malignan* OR cancer OR tumor OR neoplas* OR carcinoma) AND (hepatocellular OR liver OR hepatic OR HCC) AND (radiomics OR radiomic OR 'imaging methods') AND (prognosis OR predict* OR recurrence).

Participant or population: A total of 1929 HCC patients were taken into account.

Intervention: No intervention.

Comparator: Radiomics.

Study designs to be included: 10 studies based on radiomics were taken into account.

Eligibility criteria: (1) diagnosed with HCC by pathology, (2) identification of early recurrence of HCC by pathologic diagnosis, (3) underwent CT or MRI before hepatectomy or curative ablation, and (4) imaging analysis based on radiomics.

Information sources: Electronic database.

Main outcome(s): Radiomics is a powerful and validated tool for predicting tumor recurrence, and can provide valuable guidance for clinical decision-making in patients with HCC after surgery.

Quality assessment / Risk of bias analysis: QUADAS-2.
Strategy of data synthesis: The pooling model applied for an overall summary proportion of sensitivity and specificity rates was random (I-V heterogeneity). Forest plots were used to depict the weighted summary proportions. SROC was implemented to demonstrate the performance of the radiomic model in the included studies. Heterogeneity of the studies was evaluated by 12 statistics; heterogeneity was considered to be present if $12>50 \%$. To explore the causes of heterogeneity, we conducted a metaregression analysis of the clinical covariates. Publication bias was assessed and visually showed using Deeks' funnel plot, funnel plot asymmetry was tested by regression test. All outcomes were performed with a $95 \% \mathrm{Cl}$. Differences were considered statistically significant when the P-value was < 0.05. Stata/SE 16.0 for windows (4905 Lakeway Drive, College Station, TX 77845, USA), a meta-analysis program (https://www.stata.com) with meta-analysis packages was employed to process the statistical analyses. IBM SPSS Statistics was employed to caculate the intraclass correlation coefficient (ICC) of RQS scores.

Subgroup analysis: Because the number of studies we included is small, subgroup analysis makes no means, we didn't conduct zhe subgroup analysis.

Sensitivity analysis: The pooled sensitivity and specificity rates in predicting the early recurrence of HCC were $79 \% \quad(95 \% \mathrm{Cl}$ : $65 \%-87 \%$ ) and $83 \%$ (95\% Cl: $73 \%-90 \%$ ), respectively.

\section{Language: English.}

Country(ies) involved: China.

Keywords: Hepatocellular carcinoma, Early recurrence, Radiomics, Meta-analysis.

Contributions of each author:

Author 1 - Jiawen Yang - Project development; data collection or management; data analysis; manuscript writing; manuscript editing.

Email: 2397746531@qq.com 
Author 2 - Shuzong You - Project development; Data collection or management.

Email: yousz@enzemed.com

Author 3 - Limin Zhang - Project development; Data collection or management.

Email: 1240119941@qq.com

Author 4 - Huangqi Zhang - Data analysis.

Email: 1224190004@qq.com

Author 5 - Binhao Zhang.

Email: zhangbhao@163.com

Author 6 - Xue Dong - Manuscript editing.

Email: 17866634962@163.com

Author 7 - Wenting Pan - Manuscript

editing.

Email: yinyuyinyun@163.com

Author 8 - Shaofeng Duan - Data analysis.

Author 9 - Wenbin Ji.

Email: wb.j@163.com 